\title{
Inspection of the residual stress on welds using laser ultrasonic supported with finite element analysis
}

\author{
Chong Ye", I. Charles Ume, Yuanlai Zhou, and Vishnu V.B. Reddy \\ The Georgia W. Woodruff School of Mechanical Engineering, Georgia Institute of Technology, Atlanta, GA 30332, USA
}

Received: 5 December 2018 / Accepted: 18 January 2019

\begin{abstract}
Ultrasonic evaluation for residual stress measurement has been an effective method owing to its easy implementation, low cost and intrinsically being nondestructive. The velocity variations of acoustic waves in materials can be related to the stress state in the deformed medium by the acoustoelastic effects. In this study, a laser/EMAT ultrasonic method is proposed to evaluate the surface/subsurface longitudinal residual stress distribution generated by gas metal arc welding (GMAW). The velocity variation $\Delta V / V$ of Rayleigh wave, which is a surface wave, will be experimentally measured. Q-Switched Nd:YAG laser is used to generate a broadband ultrasonic wave. An electromagnetic acoustic transducer (EMAT) is attached to the welding plate for Rayleigh wave pick up. As the ultrasound receiver, the EMAT is used to measure time of flight (ToF) of the Rayleigh waves traveling along a specific path parallel to the direction of the welding seam. ToF measurements are obtained by changing Rayleigh wave path to welding zone center distance from 0 to $45 \mathrm{~mm}$. A $3 \mathrm{D}$ thermomechanical-coupled finite element model is then developed to validate the capability of the proposed technique for welding-induced residual stress evaluation. The distributions of the normalized velocity variations from ToF experiments are compared with the distribution of the normalized longitudinal residual stresses from finite element analysis (FEA). It has been shown that there is a good correlation between these two distributions. The proposed technique provides a potential nondestructive avenue for surface/subsurface residual stress evaluation for welding parts.
\end{abstract}

Keywords: Nondestructive testing / residual stress / laser ultrasonics / electromagnetic acoustic transducer / finite element analysis

\section{Introduction}

Residual stress generated from various manufacturing processes would influence the mechanical reliability, fatigue life, and chemical corrosion resistance of engineering structures [1-3]. Therefore, it is crucially important to precisely characterize the residual stress generation for the manufacturing components. In a typical manufacturing process, the residual stress generation could be mainly due to three reasons: the un-uniform plastic deformation, the temperature gradient-induced nonuniform material thermal expansion, and the material volume change due to material phase transformation [4]. For a typical welding process, the sharp temperature change would directly result in the nonuniform material volume change due to the large temperature gradient in the welding zone. Thus, the localized cooling and heating are the main sources introducing the residual stresses in welding. Welding is intrinsically a material melting and solidification process.

\footnotetext{
* e-mail: chongtt.ye@gmail.com
}

Therefore, the material phase transformation would also occur. Undesired residual stress in the welding structure would be detrimental to the component fatigue life and dimensional accuracy. For example, large tensile residual stress on the welds would significantly reduce the structure fatigue life. Large magnitude of residual stress would result in material plastic deformation, especially in the large-scale welding structures with strict geometrical dimensional tolerance. The in-depth understanding of residual stress generation in the welding structures could help to design and optimize the welding process parameters to ensure manufacturing reliability.

Extensive efforts have been made to experimentally measure the residual stress for manufacturing component, which includes center-hole drilling [5], ring core method [6], X-ray diffraction (XRD) $[7,8]$, and ultrasonic- $[9,10]$ and magnetic-based [11] techniques. Center-hole drilling method has been a main stream technique to evaluate the residual stress distribution in welding structures. However, a material removal procedure would be involved, which could damage the structure integrity. Therefore, it is destructive and greatly limits its application for critical 
components on field evaluation, even though it is relatively cost-effective. Ring core method can evaluate stress up to $5-7 \mathrm{~mm}$ into the specimen, deeper than a hole-drilling method. However, it causes more damage to the sample. XRD techniques are nondestructive but are only limited to residual stress measurement on the surface. Additionally, appropriate polishing and sample preparation steps are needed. The magnetic-based residual stress measurement characterizes the material residual stress by applying a magnetic field to the material. The resultant magnetic penetration will be evaluated to calculate the material residual stress states. Therefore, the magnetic-based method limits itself for the ferromagnetic materials application.

Different from the above-mentioned methods, the ultrasonic-based residual stress measurement method measures the travelling velocity of the ultrasonic waves in the material to determine the material residuals stress. The ultrasonic wave has much larger penetration depth than the XRD method. The ultrasonic methods are based on the material acoustoelastic effect, which means the residual stress states could influence the wave velocity in the material. There has been some reported research work showing the potential to predict residual stress using longitudinal waves [12], longitudinal refracted (LCR) waves [13], and shear waves [14]. For the average stress measurement in the specimen thickness direction, the bulk wave method has been very effective. However, for most of the welding components or structures, the large magnitude of tensile residual stress on the surface is the main root cause for surface crack failures. Surface waves, which propagate along the surface of the medium, have the potential to probe surface/subsurface stress, and are thus more favorable in the research reported here. In this work, Rayleigh wave is selected for investigation because the radiation loss of Rayleigh waves is minimal. In addition to its low radiation loss, the Rayleigh wave penetration depth into the specimen is only one wavelength, which makes it a superior option for surface/subsurface measurement of residual stress.

Extensive research work has been devoted for the surface wave propagation. The original surface wave propagation theory in deformed solids with uniform stress states was proposed by Hayes and Rivlin [10]. Iwashimizu extended this theory to consider propagation along arbitrary direction, not necessarily coincident to a principal direction [15]. In a later study by Hirao [16], the theory was extended to nonuniform initial stress distribution application. In the nonuniform stress states, the Rayleigh wave dispersion has been observed. The acoustoelastic effect has been successfully applied for the stress evaluation in various isotropic engineering structures. Similar research has also been carried out for the surface wave propagation in anisotropic material $[17,18]$. In a recent research conducted by Zhan et al. [19], the laser ultrasonic combined with a laser Droppler vibrometer was used to measure the welding-induced residual stress in titanium alloy.

There have been reported studies where the Rayleigh waves were used for surface residual stress evaluation with traditional ultrasonic-based residual stress measurement method [20,21]. Transmitters and receivers are required to emit and collect signals. However, high-quality interface between the transducers and the specimen is important for the accuracy of the measurement. Thus, liquid coolant is usually used in tradition to ultrasonic technique. Therefore, the development of noncontact ultrasonic technique is desired. EMAT is superior to the transducer used in traditional ultrasonic method since it does not need direct contact with the surface of the specimen. In this study, a laser/EMAT ultrasonic technique (LEU) without direct contact with the measurement structure is introduced. The EAMT collects the Rayleigh wave signals generated from the pulsed laser. Compared to the traditional ultrasonic methods, the LEU does not involve direct contact with the measurement structure, which makes it suitable for severe environment application, such as high temperature, fast moving surface, and rough surface structures. The laser/ EMAT has been a very promising technique for welding inspection since early 1990s. In the work of Johnson and Carlson [22], the laser/EMAT was used to detect flaws in the welding zone by analyzing the received wave signal. In the work of Oursler and Wagner [23], the laser/EMAT was used for the potential critical crack detection by analyzing shear waves. Different from what has been discussed in the previous papers, the laser/EMAT for the surface/subsurface residual stress emulation in the welding zone was implemented in this study. The laser/EMAT system for welding inspection is not new. The residual stress characterization with laser ultrasonic has been done by previous research. However, to the best knowledge of the authors, the utilization of the laser/EMAT for weldinginduced surface/subsurface residual stress evaluation based on the Rayleigh wave, time of flight (ToF) measurement has never been done before. Compared to the technique by Wang and Feng [24], using traditional ultrasonic detection system, EMAT is used in this study, which does not require any contact with the inspected sample. In addition, this method does not require a complicated wave signal processing, which could significantly improve the measurement reliability. Different from the research work of Dixon et al. [25] in using laser/EMAT for welding inspection without any quantitative measurement, we are using the laser/EMAT for the residual stress measurement, which imposes significantly more challenges from system setup, measurement accuracy, and data analyzing. Based on a previous study on welding residual stress calculation [26], a thermal/mechanical residual stress prediction model has been developed in this study for the residual stress prediction in the welding zone. The prediction data show a good approximation to the experimental measurement.

Welding simulation by finite element analysis (FEA) is attractive for the evaluation of the stress distribution in welded structures. The first 2D thermomechanical model for the welding analysis was proposed by Hibbitt and Marcal [27]. With the development of computational capacity, 3D model has been conducted with commercial software for the evaluation of transient temperature field and residual stress distribution [28]. In this paper, a 3D thermomechanical-coupled model has been proposed using ANSYS for welding-induced residual stress evaluation. The 


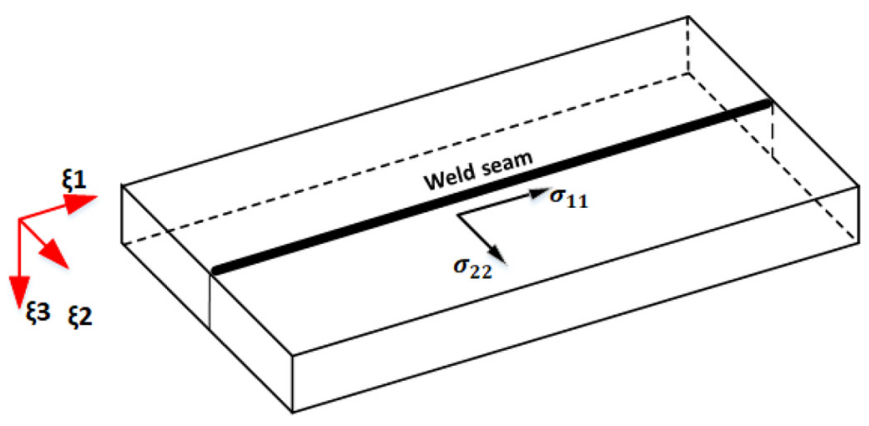

Fig. 1. Schematic diagram showing welding of two steel plates.

results show that the numerical predictions using FEA correlates well with experimental results.

\section{Theory of ultrasonic measurement}

The residual stress measurement method with ultrasonic is based on the acoustoelastic effect. In the welds, the generated residual stress would change the Rayleigh wave travelling velocity, which corresponds to different material stress states. In this experiment, the sample is prepared by welding two steel plates with GMAW technique, as shown in Figure 1. Rayleigh waves propagating only in the $\xi_{1}$ direction (the weld seam direction) are collected by a transducer. It is assumed that the Rayleigh waves could freely propagate on the welding plate surface. For simplification, a plane stress condition is assumed where the stress in the plate thickness direction $\xi_{3}$ is zero. So, only two stress components, $\sigma_{11}$ in the longitudinal direction $\xi_{1}$ and $\sigma_{22}$ in the transverse direction $\xi_{2}$, are considered. This assumption is valid based on the free boundary conditions on the thickness direction of the welded steel plate. In this study, only the principal stress components $\sigma_{11}$ and $\sigma_{22}$ are considered because the Rayleigh wave velocity change is assumed to be independent of the material shear stress.

The dependency of acoustic wave velocity on two principal state cases has been derived by Tekriwal and Mazumder [28]. The velocity variation of Rayleigh waves propagating along $\xi_{1}$ direction on the free surface $\left(\xi_{1}-\xi_{2}\right.$ plane) of an isotropic material could be related to the biaxial stress state in the following equation:

$$
\frac{\Delta V_{\mathrm{R}}}{V_{\mathrm{R}}}=\beta_{1} \sigma_{11}+\beta_{2} \sigma_{22},
$$

where $V_{\mathrm{R}}$ is the Rayleigh wave velocity, $\Delta V_{\mathrm{R}}$ is the change of Rayleigh wave velocity between stress-free solid and stressed medium. $\beta_{1}$ and $\beta_{2}$ are acoustoelastic constants in the longitudinal and transverse directions. This equation provides the Rayleigh wave velocity variation in stressed solids with two material-based constants. As also noted from this equation, the shear stress effect on the Rayleigh wave propagation in thin welding plates is ignored.

According to a previous research work [26], the weldinginduced residual stress $\sigma_{11}$ in the longitudinal direction is much larger than that of transverse direction stress $\sigma_{22}$. Additionally, the material coefficient $\beta_{2}$ is smaller than $\beta_{1}$ if the propagation direction is along $\xi_{1}$. Therefore, Rayleigh wave velocity variation induced by the transverse direction stress component is negligible. In this case, the variations of the propagating velocity of Rayleigh waves shown in equation (1) can be simplified and expressed as follows:

$$
\Delta V_{\mathrm{R}} / V_{\mathrm{R}}^{\mathrm{O}}=A \sigma_{11},
$$

where $\Delta V_{\mathrm{R}}$ is the variation of Rayleigh wave velocity and $\Delta V_{\mathrm{R}}=V_{\mathrm{R}}^{\mathrm{O}}-V_{\mathrm{R}} \cdot V_{\mathrm{R}}^{\mathrm{O}}$ is the Rayleigh wave velocity in stress-free area. $A$ is material-dependent acoustoelastic coefficient, which could be determined by the material second-order and third-order elastic constant coefficients. $\Delta V_{\mathrm{R}} / V_{\mathrm{R}}^{\mathrm{O}}$ denotes the relative variation of the propagating velocity of Rayleigh waves.

From equation (2), it is easy to say that the increase in wave velocity indicates tensile residual stress state and decrease denotes compressive stress state. However, it is hard to measure Rayleigh wave velocity directly. Alternatively, we can measure the Rayleigh wave ToF when it propagates to a specific distance. If we fix the distance to be $d_{0}$, the relative variation of Rayleigh wave velocity $\Delta V_{\mathrm{R}} / V_{\mathrm{R}}^{\mathrm{O}}$ corresponds to the relative variation of $\mathrm{ToF}$ $\Delta t_{\mathrm{R}} / t_{\mathrm{R}}^{\mathrm{O}}$. Therefore, the $\mathrm{ToF}$ variations can be related to stress states as follows:

$$
\Delta t_{\mathrm{R}} / t_{\mathrm{R}}^{\mathrm{O}}=A \sigma_{11}
$$

where

$$
\Delta t_{\mathrm{R}}=t_{\mathrm{R}}-t_{\mathrm{R}}^{\mathrm{O}}
$$

$t_{\mathrm{R}}^{\circ}$ denotes ToF of Rayleigh waves traveling $d_{0}$ in $\xi_{1}$ direction of stress-free steel plate and $t_{\mathrm{R}}$ in welding zone.

From equations (1) and (2), Rayleigh wave velocity change is linearly dependent on the magnitude of the residual stress state along the welder seam direction. From the experimental measurement, the ToF for Rayleigh wave traveling along distance $d_{0}$ is directly measured by the EMAT. Acoustoelastic constant $A$ links the velocity variation and the longitudinal residual stress. It depends on material properties and could be calibrated using INSTRON for uniaxial tensile test combined with the proposed laser ultrasonic technique. However, calibration was not carried out in this research work due to instrumental limitations. As a result, the actual residual stress could not be obtained in the absence of acoustoelastic constant. Alternatively, normalization is used during data analysis for the validation of the accuracy of the proposed laser/EMAT technique.

A normalization procedure is implemented by dividing the velocity measurement data with the maximum value. The welding-induced residual stress of the welder could be obtained from the resulting velocity variation distribution of the normalized Rayleigh waves.

\section{Finite element analysis}

A $114.3 \times 140 \times 12.5 \mathrm{~mm}^{3}$ low carbon steel plate was modeled in ANSYS using the symmetric boundary 


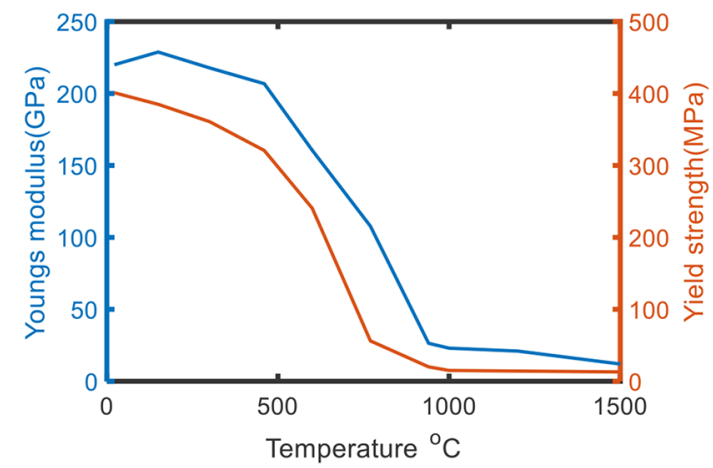

Fig. 2. The mechanical properties of low carbon steel as a function of temperature.

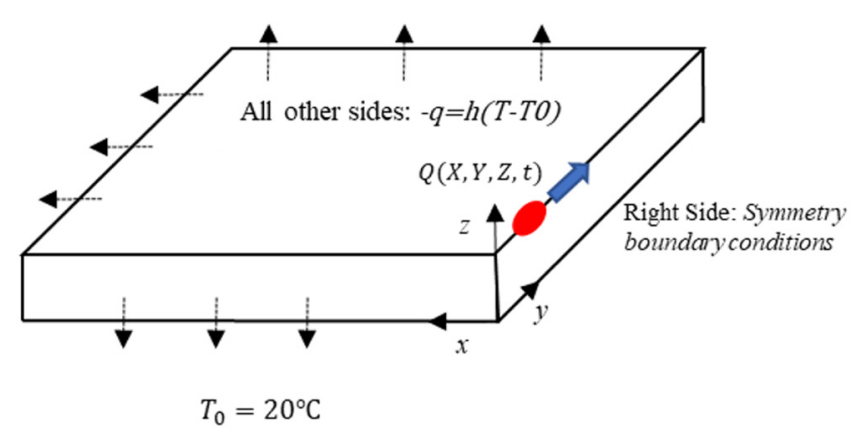

Fig. 3. The thermal boundary in the FEA model.

conditions. The mechanical properties of the low carbon steel as a function of temperature is listed in Figure 2 [29]. Both the modulus and the yield strength would significantly decrease with the increasing temperature. During simulation, the material is assumed to be elastic - perfectly plastic. The material poison's ratio is 0.29 . The thermal expansion coefficient dose not variate significantly with the temperature, and it is selected to be $1.7 \times 10^{-6} \mathrm{~K}^{-1}$. The material density is $7.2 \times 10^{3} \mathrm{~kg} / \mathrm{m}$. This model was developed with ANSYS in a two-step procedure. In the first step, heat flux of Gaussian distribution shown in equation (5) was applied as input to simulate the heat source of welding process:

$$
Q=Q_{\mathrm{m}} e^{\frac{-3\left(x^{2}+\left(y-V_{t}\right)^{2}\right)}{R^{2}}},
$$

where

$X$ and $Y$ represent the coordinates, and $Y$ direction is parallel to the weld seam.

$V$ and $R$ denote travel speed of the arc and effective weld arc radius, respectively.

$Q_{\mathrm{m}}$ is the maximum heat flux at the center and can be expressed as follows:

$$
Q_{\mathrm{m}}=\eta \frac{\partial U I}{\pi R^{2}}
$$

where $U$ and $I$ are voltage and current during welding process and $\eta$ denotes welding thermal efficiency.

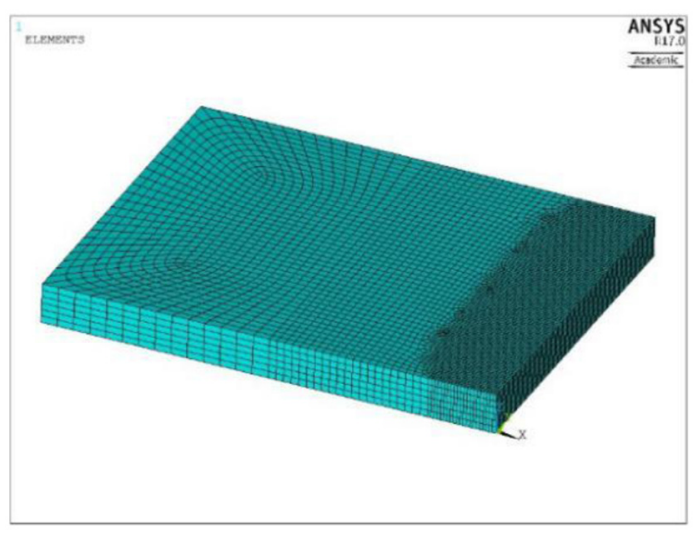

Fig. 4. Mesh of FEA model.

Convection was applied on all external boundaries and the reference/ambient temperature was considered as $20{ }^{\circ} \mathrm{C}$. The applied boundary conditions are shown in Figure 3. The four-node linear element with thermal/ mechanical coupling was selected for the analysis. The implicit algorithm was used to solve the problem.

In the next step, thermal results along with structural boundary conditions were fed to solve the structural problem to get longitudinal residual stress distribution on the surface of the welded plates.

Mesh convergence analysis was carried out, and an element size of $1.25 \mathrm{~mm}$ was found to give optimal solution with reasonable computational time. Mesh is shown in Figure 4. Both transient thermal and transient structural analysis were carried out in four time steps. During the first time step, which was the simulated welding process, 48 substeps of $2 \mathrm{~s}$ per substep were considered. And then substep size was increased to $10 \mathrm{~s}$ gradually to reduce the computational time. Temperature distribution at $t=5 \mathrm{~s}$ and $t=1200 \mathrm{~s}$ are shown in Figures $5 \mathrm{a}$ and $5 \mathrm{~b}$. Since the maximum value of color bar in Figure $5 \mathrm{a}$ is around $1344^{\circ} \mathrm{C}$, the temperature of the welding center, which is beyond $1344^{\circ} \mathrm{C}$, is not shown. Thermal solution data were saved at every substep and then retrieved during the structural loading. Temperature distribution at the end of $1200 \mathrm{~s}$ is uniform and very close to ambient temperature. Therefore, the stress at the end of $1200 \mathrm{~s}$ was regarded as the residual stress, which is shown in Figure 6.

After normalization, the distribution of simulated residual stress for all the corresponding test rows is shown in Figure 7. Each test row is a selected residual stress sampling line that is normal to the welding direction.

\section{Experiment}

\subsection{Sample description}

For the FEA model validation, the same size low carbon steel plates containing a single pass butt weld over their center produced by GMAW were prepared. The sample was joined by a Miller Pulstar 450 GMAW machine. The weld gun was controlled by a General Electric P50 process robot. The welding voltage was $25 \mathrm{~V}$ and the welding speed 


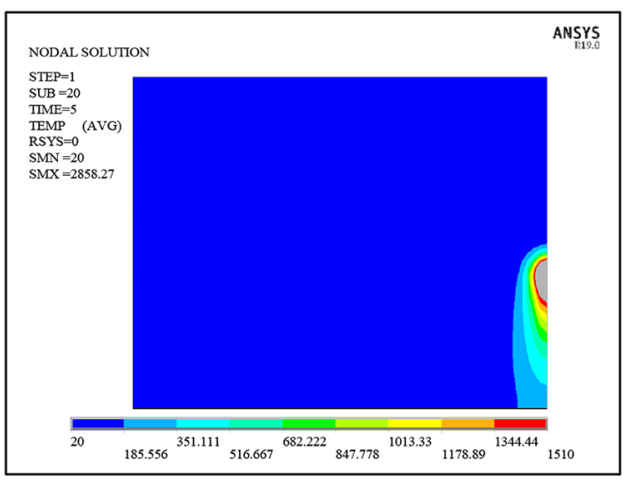

(a)

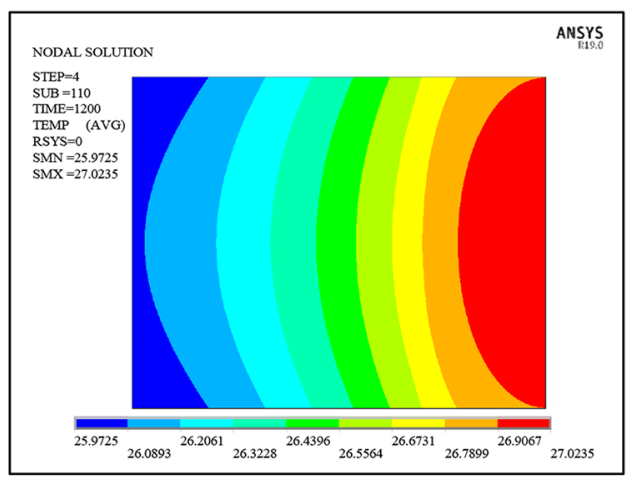

(b)

Fig. 5. (a) Temperature distribution at $5 \mathrm{~s}$ (during the welding process. Units $\left.{ }^{\circ} \mathrm{C}\right)$. (b) Temperature distribution at $1200 \mathrm{~s}(11 \mathrm{~min} 48 \mathrm{~s}$ after the welding process stopped. Unit: $\left.{ }^{\circ} \mathrm{C}\right)$.

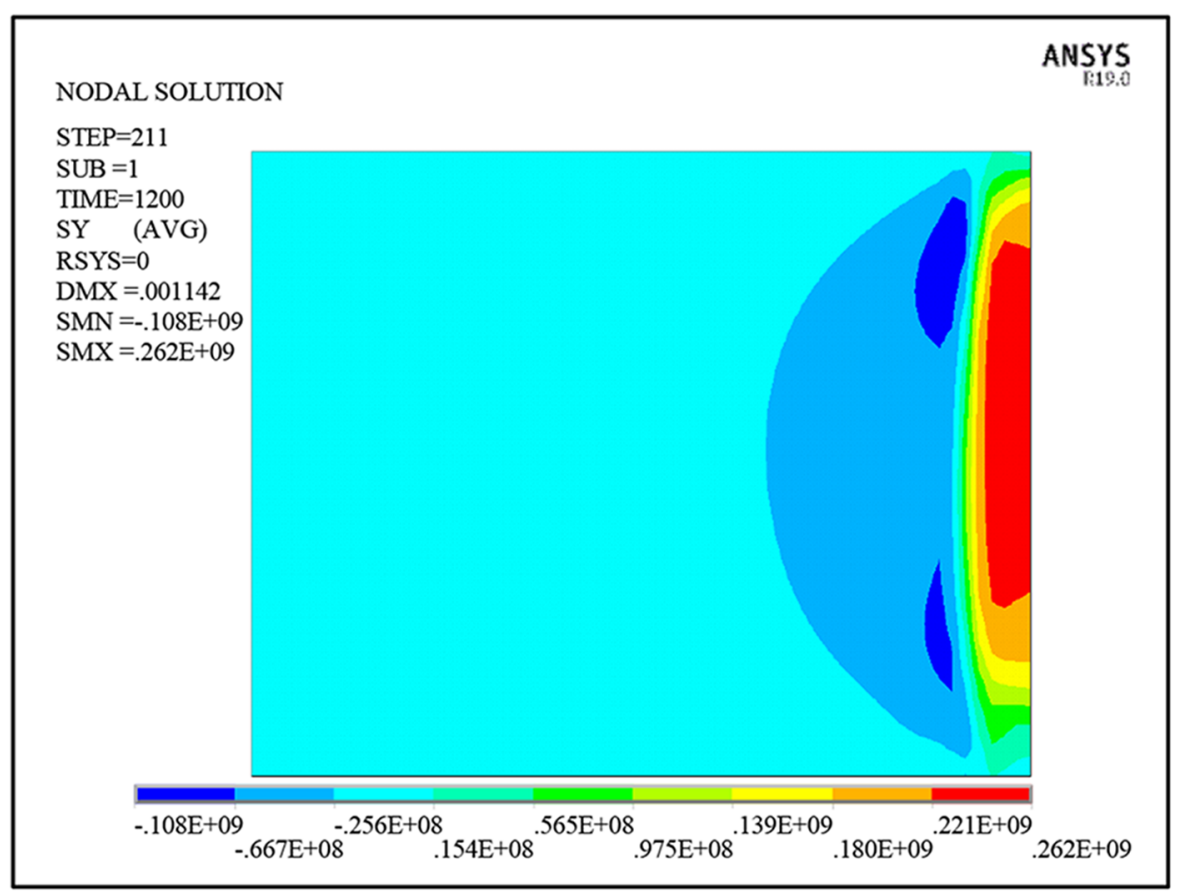

Fig. 6. Welding-induced longitudinal stress (Unit: MPa).

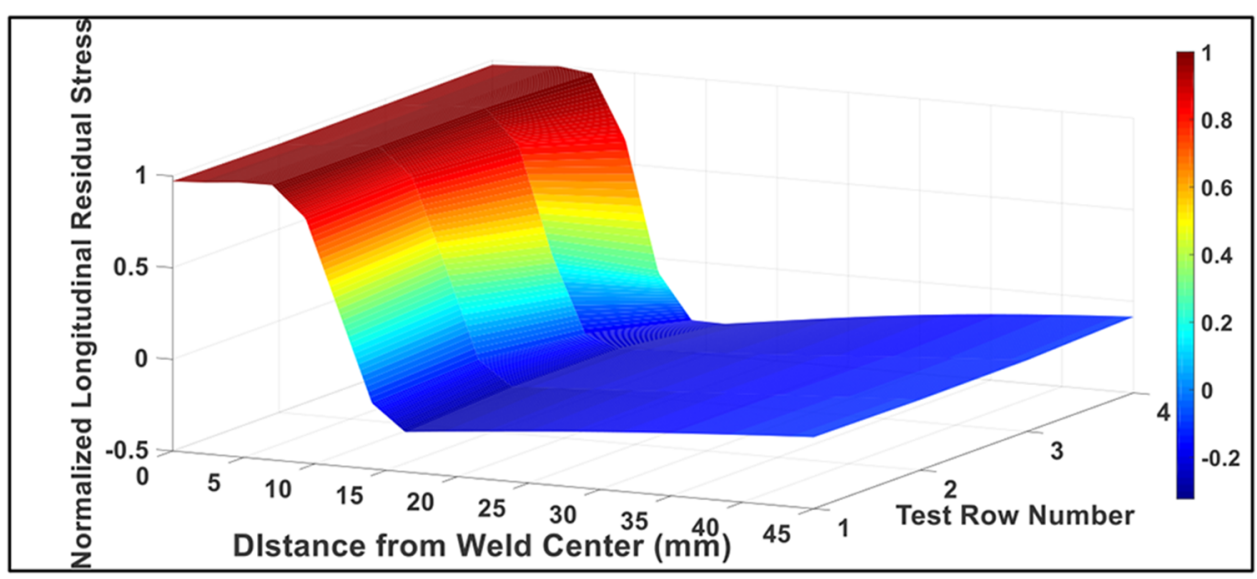

Fig. 7. Distribution of longitudinal residual stress on welded sample from simulation. 
was set as $0.375 \mathrm{in} . / \mathrm{s}$. The picture of the sample under study and the designed coordinates are shown in Figure 8. The clamps were used to fix the plates at both ends during the welding process, as shown in Figure 9. After welding, the weld butt reinforcement needs to be removed to adapt to the EMAT probe flat surface. The micro-surface grinding process was used for the reinforcement of part removal. The depth of cut in each grinding step was set to

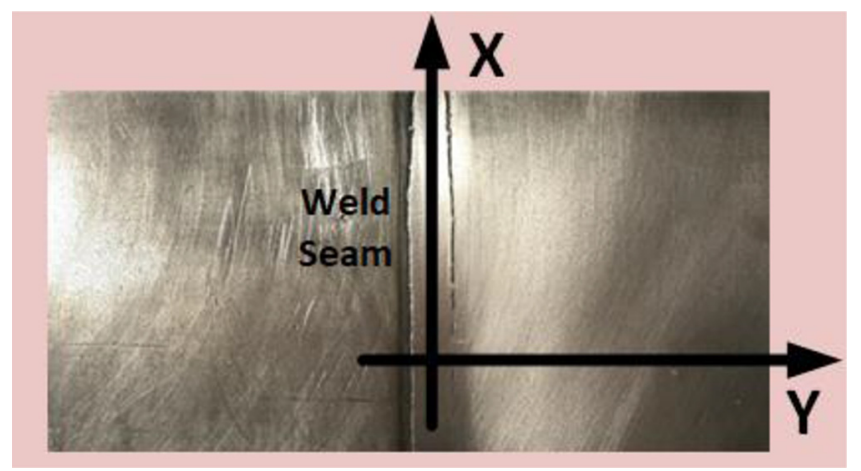

Fig. 8. Welded sample.

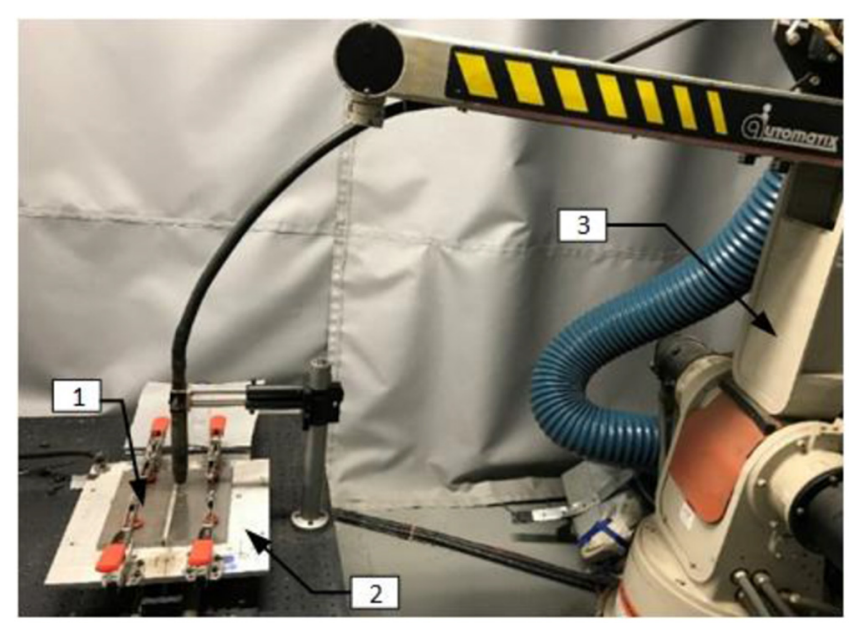

Fig. 9. Welding setup (1 - sample, 2 - positioning system, 3 - welding robot). be $15 \mu \mathrm{m}$ to avoid any residual stress introduced from grinding. Additionally, coolant was introduced in the grinding process to reduce the temperature effect on external residual stress generation. The longitudinal residual stress, which is the main residual stress developed from GMAW process, is along the weld seam as in $x$-direction.

\subsection{Experimental setup}

Figure 10 shows the laser measurement system setup, including a Nd:YAG pulsed laser, a customized EMAT, a control unit, a data acquisition system, and a twodimensional position stage driven by step motors. The schematic diagram of the measurement system is shown in Figure 11.

The pulsed Nd:YAG laser source (Continuum) was used for laser generation. The laser was focused to the surface of the sample through optic lenses. The incident point on the sample surface has a diameter of around $5 \mathrm{~mm}$. Rayleigh waves that were excited by the pulsed laser propagated along all the directions on the surface of the testing sample. However, the EMAT (BWX

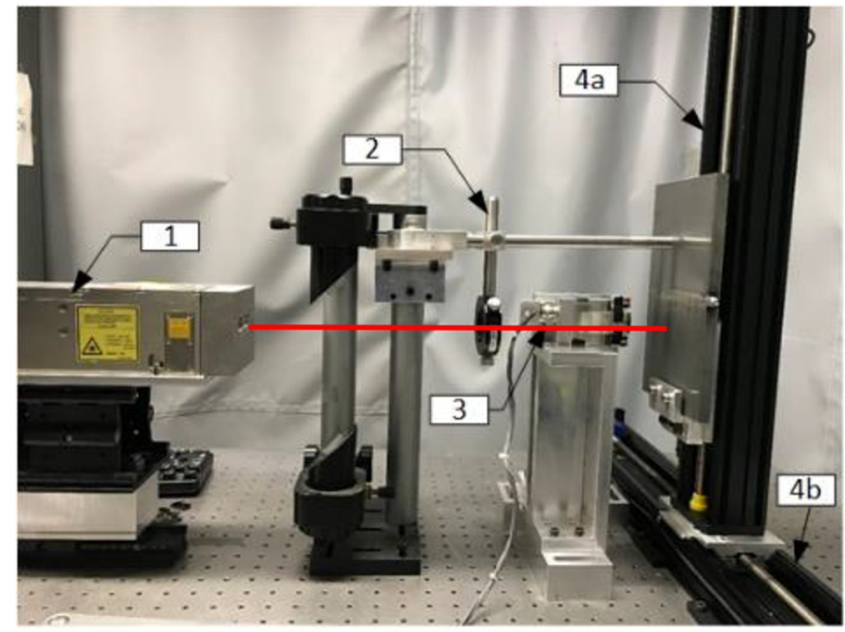

Fig. 10. Laser/EMAT stress measurement setup (1 - laser; 2 - convex lens; 3 - EMAT; $4 \mathrm{a}$ - linear stage $x ; 4 \mathrm{~b}$ - linear stage $y$ ).

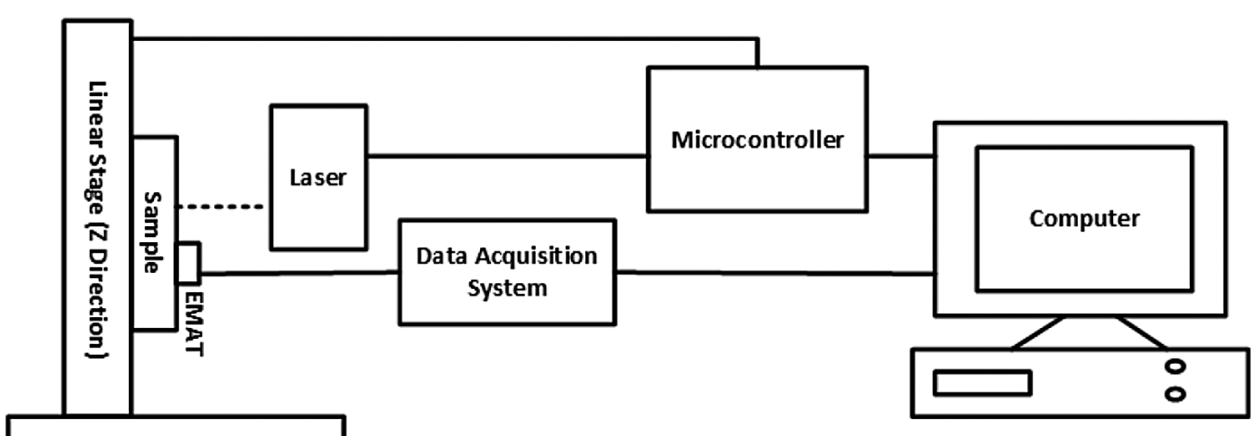

Linear Stage (X Direction)

Fig. 11. Schematic diagram of experimental system. 


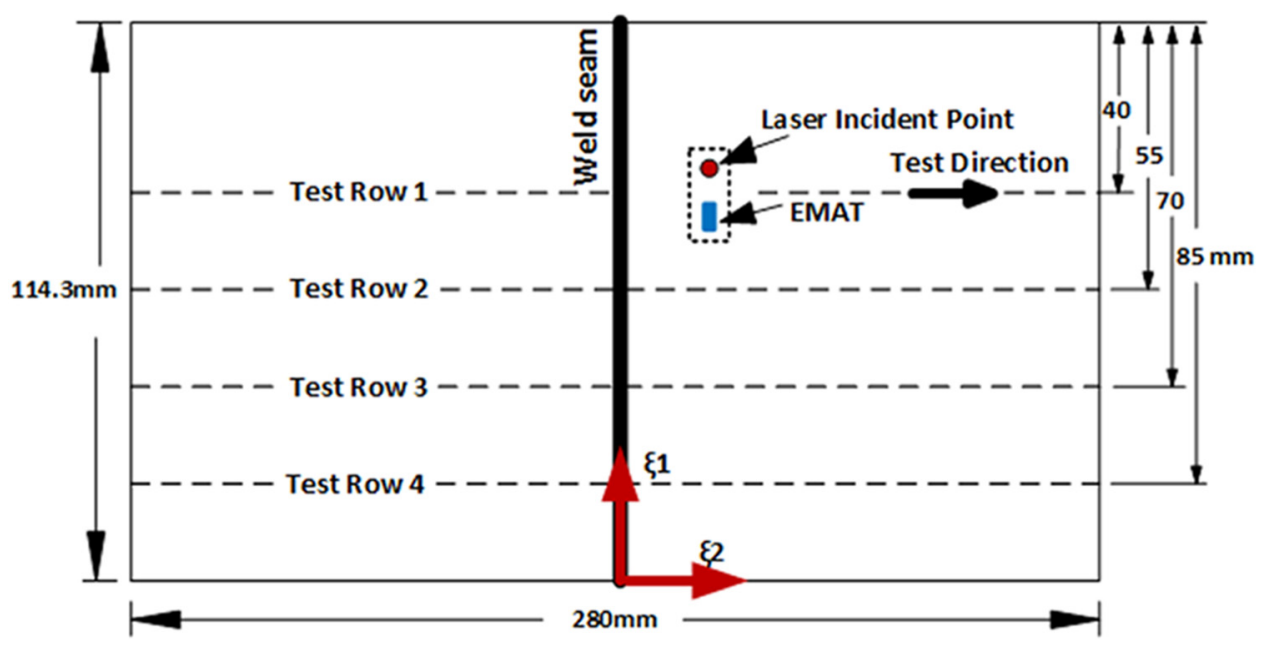

Fig. 12. Schematic diagram showing the ToF testing procedure.

Technologies, Inc.) only captured the Rayleigh waves travelling along the welding seam direction. The EMAT has a reception bandwidth of $(0.5 \mathrm{MHz}, 2.0 \mathrm{MHz})$. There are four separate coils with $2 \mathrm{~mm}$ pitch in-between. The data acquisition system consists of a high-speed fourchannel Gage Compuscope 8349 PCI/AD card.

The two-dimensional translation stage consists of two linear stages placed normal to each other, with one point to $x$-direction, and the other one along $z$-direction. Both stages have a positional resolution of $25 \mu \mathrm{m} / \mathrm{step}$. The microcontroller was used to control the two-dimensional translation stage movement. A MATLAB script was used to power up the laser and control-positioning stage movement. The laser system has a time resolution of $1 \mathrm{~ns}$ to enable the accuracy of ToF measurements. The whole measurement system was automated and controlled by a MATLAB GUI on a PC.

The distance between the laser head and EMAT was kept constant at $11 \mathrm{~mm}$ during the ToF measurement. The whole system was placed on a vibration table to avoid vibration-induced errors. The testing sample was clamped onto the linear stages. The wheels of the EMAT help to make it roll on the surface of the sample when the sample moves with the linear stages.

\subsection{Time of flight measurements}

ToF measurements have been widely used in acoustic wave measurement to determine the time required to travel a certain distance in a medium. The nondestructive testing for defects or stress assessment employ a wide variety of ToF measurements $[29,30]$. In the measurements, waves travel along a predetermined distance and the arriving time of the signal of interest is recorded. To measure the residual stress on the welding plate surface, the ToF of near surface Rayleigh waves were measured. The reference time $t_{0}$ was obtained by measuring the ToF on a stress-free low carbon steel sample. To get the residual stress distribution on the welder, the ToF of Rayleigh waves were measured along different points normal to the weld seam direction.
In order to evaluate stress states of a larger area, four inspection rows normal to the weld seam direction with a pitch of $15 \mathrm{~mm}$ were selected, as shown in Figure 12. The welding residual stress evaluation area covers any location that is $45 \mathrm{~mm}$ away from the weld seam. Along each inspection row, 15 equally spaced points between 0 and $45 \mathrm{~mm}$ away from the welding seam were chosen for the residual stress evaluation. The welding-induced residual stress distribution normal to the welding seam could be obtained. In each measurement location, the ToF measurements were repeated 300 times and the average was used to achieve better signalto-noise ratio (SNR). A typical Rayleigh wave amplitude signal by averaging 300 repeated measurements is shown in Figure 13. The Rayleigh wave has a bipolar pulse as shown in the figure, which corresponds to the maximum amplitude of the wave without radiation loss. The total time $t$ that the Rayleigh wave travels through is taken as the ToF. The B-spline curve interpolation method was implemented here to interpolate the Rayleigh wave amplitude to achieve a time resolution less than $1 \mathrm{~ns}$.

The normalized distribution of relative velocity variations on 15 locations with varying distance from weld center is shown in Figure 14. The 3D distribution also shows the difference between each test row.

\section{Results and discussion}

From ToF measurements, longitudinal residual stresses of four test rows on the welded sample were obtained. On each of the four measurement rows, the stress states were measured at 15 points with different distances away from the weld seam center. To get the true residual stress value, the acoustoelastic coefficient needs to be calibrated by the proposed technique together with Instron. In this study, the residual stress is normalized due to the lack of acoustoelastic coefficient. The normalized residual stress distribution as a function away from the welding seam was 


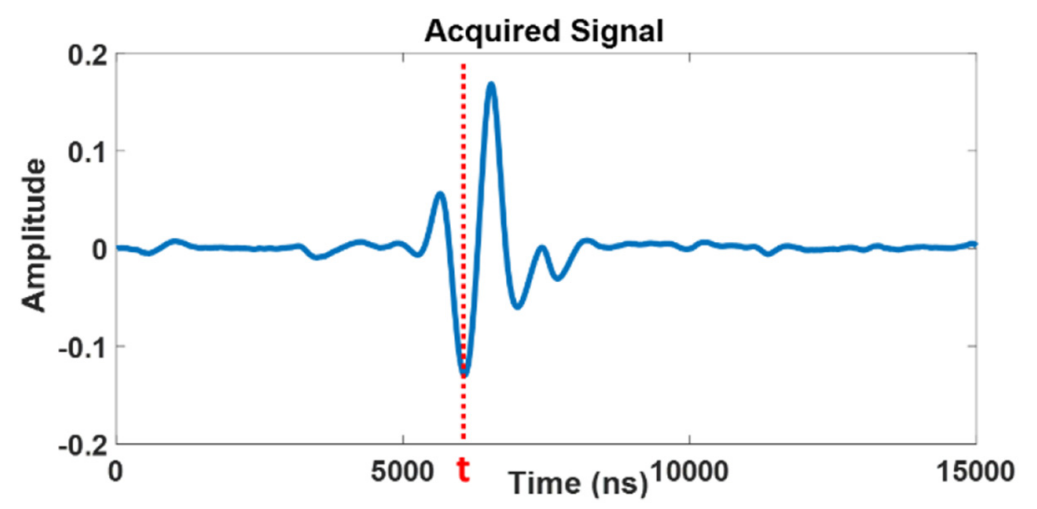

Fig. 13. Rayleigh wave average signal by EMAT.

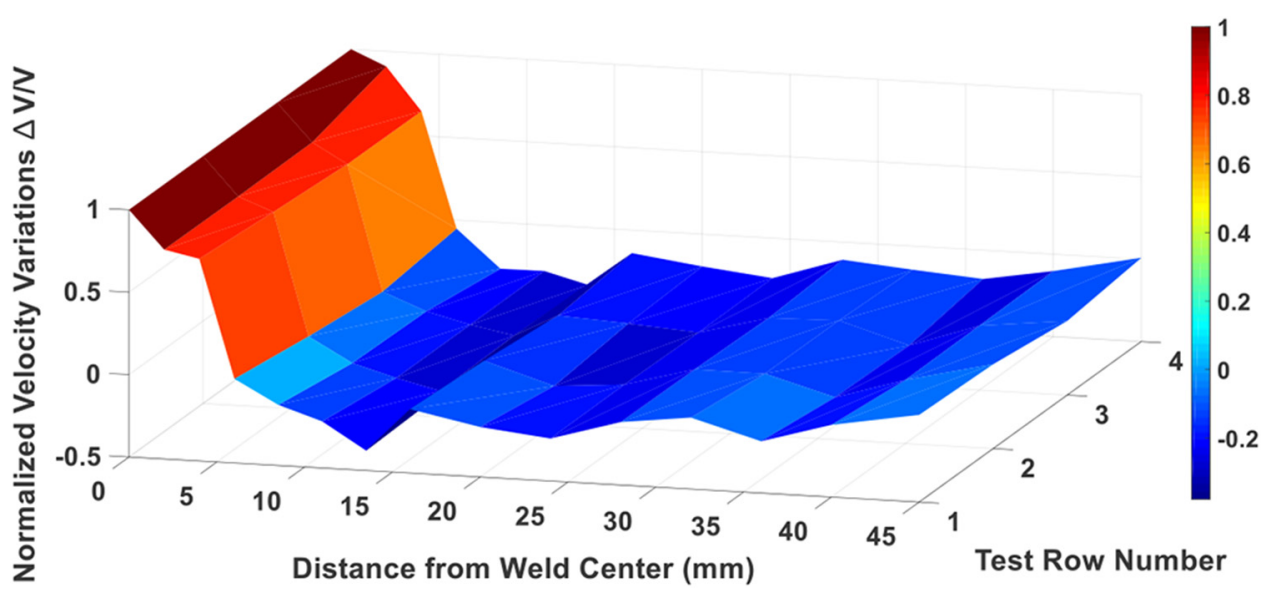

Fig. 14. Distribution of longitudinal residual stress on welded sample from ToF measurements.

also evaluated from FEA. The residual stress distribution comparisons from the ToF measurement and FEA calculations are plotted against each other, as shown in Figure 15.

From equation (1), the longitudinal residual stress after welding process is proportional to the relative variations of the phase velocity of Rayleigh waves propagating along the weld seam. Theoretically, the normalized longitudinal stress distribution should be the same as the normalized velocity variation profile. Figure 14 shows the comparison between these two profiles, and there is a good correlation between them with an acceptable small deviation. The reasons for these small deviations are as follows:

- The residual stress generation in welding could come from temperature gradient-induced material nonuniform volume change and phase transformation-induced material volume change. As noted in this work, material melting and solidification during the welding process is not considered in the simulation.

- Rayleigh wave velocity depends on the stress state within its propagation region, which is about one wavelength deep into the sample. Thus, the velocity variations of Rayleigh waves reflect the average stress state of its propagation region. However, simulation evaluates only surface stress state.

Both the experimental and simulation results show that the biggest tensile stress appears in the weld seam, which is in the melted zone. As the distance away from the weld seam, the large magnitude of tensile residual stress sharply decreases and changes to compressive one at the edge of the heat-affected zone. The large magnitude of the tensile stress in the center of melting zone comes from the material volume shrinkage after the welding during the cooling down process. At the high temperature, the plate material was melted and the welding groove was filled by liquid metal. During the cooling down, the weld center has the largest temperature decrease, which results in the material shrinkage while the outer area temperature change is not significant. The fast and significant decrease of the temperature in the weld center leads to the material shrinkage of the hot metal and a tensile stress develops.

As we expected, the difference of stress distribution between each test row is not obvious. This can be explained by the temperature distribution during the welding process, which only has large gradient in the direction 


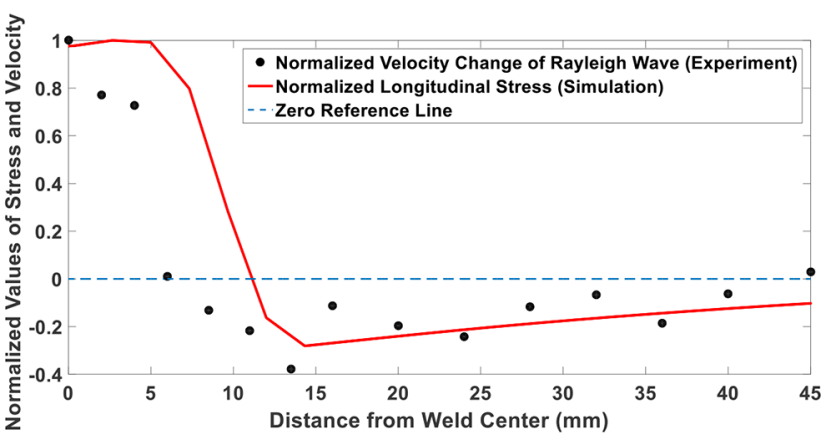

(a)

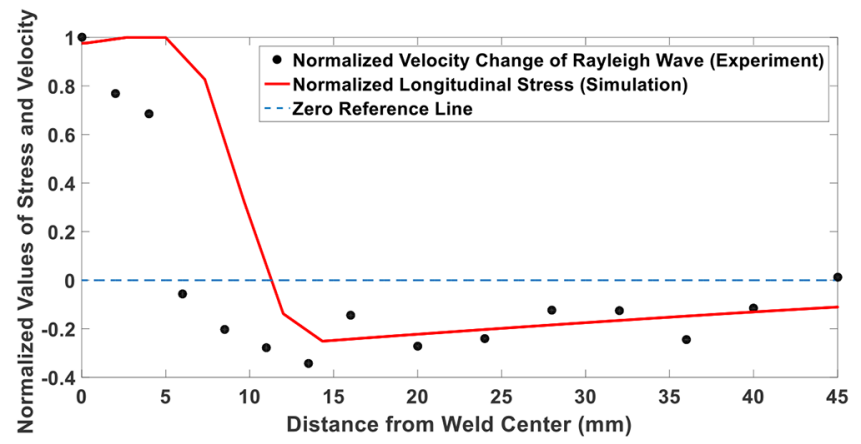

(b)

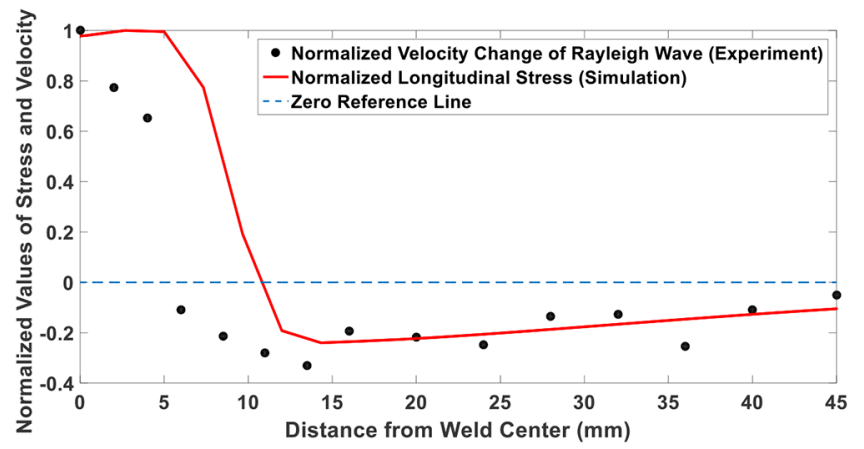

(c)

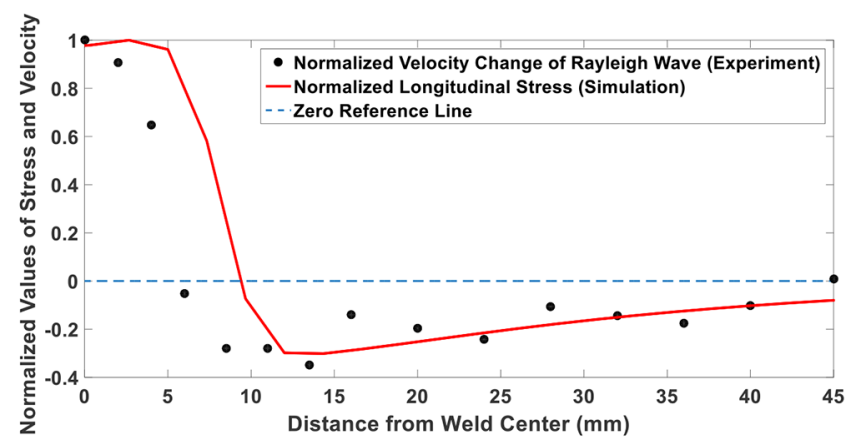

(d)

Fig. 15. Comparison of normalized relative velocity variations from ToF experiments and normalized longitudinal residual stress from FEA simulation for (a) row \# 1 tests, (b) row \#2 tests, (c) row \#3 tests, and (d) row \#4 tests.

normal to the weld seam. As a result, the magnitude of welding-induced residual stress is mainly dependent on the distance to the weld seam center. That is to say, the stress states of the inspection points are identical if the distances of those inspection points to the weld seam center are the same.

The residual stress generation in the welding zone is a very complicated process, which involves the thermal, mechanical and material phase transformation. A comprehensive residual stress prediction for welding needs to include the uniform material deformation, temperature gradient-induced nonuniform material volume change, and material phase transformation-induced volume change. The temperature gradient-induced nonuniform material volume change is the dominating factor contributing to the residual stress generation in welding. So, in this study, only the temperature effect is considered in residual stress prediction. In terms of the experimental measurement, we are based on acoustoelastic effect. Assumptions are made and we consider only the effect of longitudinal residual stress on wave velocity change and ignore the effect of transverse residual stress. However, in cases where transverse residual stresses are large and cannot be ignored, this assumption might introduce errors. Other measurement errors can also come from ToF measurement of Rayleigh waves, resulting in the error of Rayleigh wave velocity.

The Rayleigh wave can only penetrate to around one wave length distance into the material. So, the proposed method is limited to the surface residual stress measurement. To measure the residual stress underneath the surface, appropriate surface removal procedure needs to be implemented, such as electrochemical polishing.

\section{Conclusions}

An ultrasonic-based laser/EMAT method is developed in this study for the welding-induced residual stress measurement with high accuracy. The method utilizes a high-precision EMAT to measure the ToF of the Rayleigh waves traveling along the welding plate surface induced by the laser pulse. The linear relationship between the Rayleigh wave velocity variation along the welding seam direction and the residual stress states has been developed. The experimental measurement is conducted by measuring the normalized residual stress distribution normal to the welding seam direction from the ToF measurements. A thermal-mechanical coupled FEA model was developed for the residual stress prediction during the metal welding process. A good match is found between the experimental measurement and FEA model calculation. The laser/EMAT for residual stress measurement could be used for machining-induced residual stress measurement as well as measurement associated with residual stress development in metal additive manufacturing. Being also noted in this research, an EMAT with a flat surface would need to be attached to the sample in order to receive the acoustic signal. Therefore, the measurement sample needs to be well polished to have a surface that is sufficiently flat. Regardless, the proposed FEA model and intrinsically nondestructive and noncontact laser/ EMAT method provide a useful means for residual stress evaluation for various engineering welding structures. 


\section{Author contribution statement}

Conceptualization: Chong Ye; formal analysis: Chong Ye; investigation: Yuanlai Zhou; methodology: Chong Ye and Yuanlai Zhou; resources: Charles Ume; supervision: Charles Ume; validation: Chong Ye and Vishnu V.B. Reddy; writing - original draft: Chong Ye; writing review and editing: Charles Ume.

\section{Funding}

This research received no external funding.

\section{Conflicts of interest}

The authors declare no conflict of interest.

\section{References}

1. D. Radaj, Welding residual stress and distortion, Heat Effects of Welding, Springer, Berlin, 1992, pp. 129-246

2. Z. Pan et al., Turning induced residual stress prediction of AISI 4130 considering dynamic recrystallization, Mach. Sci. Technol. 22 (2018) 507-521

3. D. Deng, FEM prediction of welding residual stress and distortion in carbon steel considering phase transformation effects, Mater. Des. 30 (2009) 359-366

4. Z. Pan, S.Y. Liang, H. Garmestani, Finite element simulation of residual stress in machining of Ti-6Al-4V with a microstructural consideration, Proc. Inst. Mech. Eng. B: J. Eng. Manuf. 2018. DOI: doi.org/10.1177/0954405418769927

5. G. Schajer, Measurement of non-uniform residual stresses using the hole-drilling method. Part I: stress calculation procedures, J. Eng. Mater. Technol. 110 (1988) 338-343

6. A. Giri, M. Mahapatra, On the measurement of sub-surface residual stresses in SS 304L welds by dry ring core technique, Measurement 106 (2017) 152-160

7. P.S. Prevey, X-ray diffraction residual stress techniques, Metals Handbook, American Society for Metals, Ohio, 1986, pp. $380-392$

8. A. Allen et al., Neutron diffraction methods for the study of residual stress fields, Adv. Phys. 34 (1985) 445-473

9. D. Crecraft, The measurement of applied and residual stresses in metals using ultrasonic waves, J. Sound Vib. 5 (1967) 173-192

10. M. Hayes, R.S. Rivlin, Surface waves in deformed elastic materials, Arch. Rational Mech. Anal. 8 (1961) 358

11. I.C. Noyan, J.B. Cohen, Residual stress: measurement by diffraction and interpretation, Springer, Berlin, 2013

12. A. Karabutov et al., Laser ultrasonic diagnostics of residual stress, Ultrasonics 48 (2008) 631-635
13. Y. Javadi, M. Akhlaghi, M.A. Najafabadi, Using finite element and ultrasonic method to evaluate welding longitudinal residual stress through the thickness in austenitic stainless steel plates, Mater. Des. 45 (2013) 628-642

14. R. King, C. Fortunko, Determination of in-plane residual stress states in plates using horizontally polarized shear waves, J. Appl. Phys. 54 (1983) 3027-3035

15. Y. Iwashimizu, O. Kobori, The Rayleigh wave in a finitely deformed isotropic elastic material, J. Acoust. Soc. Am. 64 (1978) 910-916

16. M. Hirao, H. Fukuoka, K. Hori, Acoustoelastic effect of Rayleigh surface wave in isotropic material, J. Appl. Mech. 48 (1981) 119-124

17. G.T. Mase, G. Johnson, An acoustoelastic theory for surface waves in anisotropic media, J. Appl. Mech. 54 (1987) $127-135$

18. J. Lothe, D. Barnett, On the existence of surface-wave solutions for anisotropic elastic half-spaces with free surface, J. Appl. Phys. 47 (1976) 428-433

19. Y. Zhan et al., Residual stress in laser welding of TC4 titanium alloy based on ultrasonic laser technology, Appl. Sci. 8 (2018) 1997

20. M. Duquennoy et al., Ultrasonic characterization of residual stresses in steel rods using a laser line source and piezoelectric transducers, NDT \& E Int. 34 (2001) 355-362

21. E. Tanala et al., Determination of near surface residual stresses on welded joints using ultrasonic methods, NDT \& E Int. 28 (1995) 83-88

22. J.A. Johnson, N.M. Carlson, A laser/EMAT concurrent weld inspection system, Review of Progress in Quantitative Nondestructive Evaluation, Springer, Berlin, 1991, pp. 2097-2104

23. D.A. Oursler, J.W. Wagner, Narrow-band hybrid pulsed laser/EMAT system for non-contact ultrasonic inspection using angled shear waves, Review of Progress in Quantitative Nondestructive Evaluation, Springer, Berlin, 1995, pp. 553-560

24. J. Wang, Q. Feng, Residual stress determination of rail tread using a laser ultrasonic technique, Laser Phys. 25 (2015) 056104

25. S. Dixon, C. Edwards, S.B. Palmer, A laser-EMAT system for ultrasonic weld inspection, Ultrasonics 37 (1999) 273-281

26. C. Ye et al., Welding induced residual stress evaluation using laser-generated Rayleigh waves, AIP Conference Proceedings, AIP Publishing, College Park, MD, 2018

27. H.D. Hibbitt, P.V. Marcal, A numerical, thermo-mechanical model for the welding and subsequent loading of a fabricated structure, Comput. Struct. 3 (1973) 1145-1174

28. P. Tekriwal, J. Mazumder, Finite element analysis of threedimensional transient heat transfer in GMA welding, Welding J. 67 (1988) 150s-156s

29. J. Chen, B. Young, B. Uy, Behavior of high strength structural steel at elevated temperatures, J. Struct. Eng. 132 (2006) 1948-1954

30. D. Stamenković, I. Vasović, Finite element analysis of residual stress in butt welding two similar plates, Sci. Tech. Rev. 59 (2009) 57-60

Cite this article as: Chong Ye, I. Charles Ume, Yuanlai Zhou, Inspection of the residual stress on welds using laser ultrasonic supported with finite element analysis, Manufacturing Rev. 6, 3 (2019) 